\title{
Adaptive thermal property control technique for holistic thermal management of mobile devices
}

\author{
Kyoung Min Lee ${ }^{1,2 *}$, Yunhyeok Im $^{1 *}$, Jun Ho Huh ${ }^{1}$, \\ and Tae Hee Han ${ }^{2 a)}$ \\ ${ }^{1}$ System LSI Division, Samsung Electronics Co. Ltd, Hwaseong 18448, Korea \\ 2 The College of Information and Communication Engineering, \\ Sungkyunkwan University, Suwon 16419, Korea \\ a)than@skku.edu
}

Abstract: From the perspective of user experience and safety when using mobile devices, skin (outer surface) temperature-aware thermal management, along with the application processor die-junction temperature, is crucial. Traditional thermal management techniques have ignored the combined effect of the junction and skin temperatures, resulting in unnecessary performance degradation due to excessive thermal throttling. We propose a novel thermal management method for mobile devices, by incorporating an adaptive thermal property control (ATPC) technique. The ATPC technique is designed to adapt the thermal properties between the junction and skin according to their thermal margins. Intensive simulation results show that the ATPC technique prolongs the maximum performance duration of the mobile device up to $34 \mathrm{~min}$ in the nominal application processor power consumption range of 2.9-4.6 W. In other words, the technique provides a performance gain of approximately $7 \%$ by preventing false early thermal throttling.

Keywords: system-on-chip, application processor, thermal management, thermal property control, thermal meta-materials

Classification: Electron devices, circuits and modules

\section{References}

[1] S. V. Garimella, et al.: "Electronics thermal management in information and communications technologies: Challenges and future directions," IEEE Trans. Compon. Packag. Manuf. Technol. 7 (2017) 1191 (DOI: 10.1109/TCPMT. 2016.2603600).

[2] E. Rotem, et al.: "Power and thermal constraints of modern system-on-a-chip computer,” Microelectronics J. 46 (2015) 1225 (DOI: 10.1016/j.mejo.2015.09. 002).

[3] H. Zhang, et al.: "Thermal sensation, rate of temperature change, and the heat dissipation design for tablet computers," Appl. Ergon. 62 (2017) 197 (DOI: 10. 1016/j.apergo.2017.03.006).

[4] M. Halpern, et al.: "Mobile CPU's rise to power: Quantifying the impact of 
generational mobile CPU design trends on performance, energy, and user satisfaction," HPCA (2016) 64 (DOI: 10.1109/HPCA.2016.7446054).

[5] J. Kong, et al.: "Recent thermal management techniques for microprocessors," ACM Comput. Surv. 44 (2012) 13 (DOI: 10.1145/2187671.2187675).

[6] M. Pedram and S. Nazarian: "Thermal modeling, analysis, and management in VLSI circuits: Principles and methods," Proc. IEEE 94 (2006) 1487 (DOI: 10.1109/JPROC.2006.879797).

[7] Intel: Intel Docea Power and Thermal Modeling and Simulation Solutions (2017) https://www.intel.com/content/www/us/en/system-modeling-andsimulation/docea/overview.html.

[8] Samsung Electronics: Exynos 9 (2017) http://www.samsung.com/ semiconductor/minisite/Exynos/Solution/MobileProcessor/Exynos_9_Series_ 8895.html.

[9] T. Zhang and T. Luo: "High-contrast, reversible thermal conductivity regulation utilizing the phase transition of polyethylene nanofibers," ACS Nano 7 (2013) 7592 (DOI: 10.1021/nn401714e).

[10] R. Zheng, et al.: "Reversible temperature regulation of electrical and thermal conductivity using liquidsolid phase transitions," Nat. Commun. 2 (2011) 289 (DOI: $10.1038 / \mathrm{ncomms} 1288$ ).

[11] M. A. Beasley, et al.: "MEMS thermal switch for spacecraft thermal control," Proc. SPIE 5344 (2004) (DOI: 10.1117/12.530906).

\section{Introduction}

Traditionally, junction temperature has been the sole metric for thermal management. However, the skin temperature of the mobile device is a critical factor for ergonomic thermal management. Fully utilizing the thermal budget of the junction and skin simultaneously is challenging, because the thermal behavior and tolerable temperature ranges are different between the junction and skin [1]. In terms of the tolerable temperature range, the allowed maximum junction temperature is approximately $85^{\circ} \mathrm{C}$, considering reliability stress, and the skin temperature limit ranges between $40^{\circ} \mathrm{C}$ and $45^{\circ} \mathrm{C}$ for comfortable use $[1,2,3]$. The typical thermal response time of the junction temperature is a few microseconds, whereas that of the skin temperature is up to a minute [1]. For these reasons, the thermally critical region varies with the power consumption level of the application processor (AP), which is the dominant heat source of the mobile device [4]. When the AP power consumption is relatively low, thermal throttling is not required, as shown in Fig. 1(a). A gradual increase of AP power consumption makes the skin temperature as the dominant factor for thermal throttling, as shown in Fig. 1(b). Conversely, in the high-power use cases, such as high-definition video recording or playing, the junction temperature acts as the critical factor. This is because the junction temperature rises drastically, whereas the skin does not experience any significant change over a short period, owing to the heat capacity difference between the junction and skin, as shown in Fig. 1(c).

Conventional thermal management strategies cannot cope with the thermal budget unbalancing, because the thermal properties such as thermal conductivity, heat capacity, and density in the system are handled as fixed factors $[1,5]$. We present an adaptive thermal property control (ATPC) technique that can utilize the 


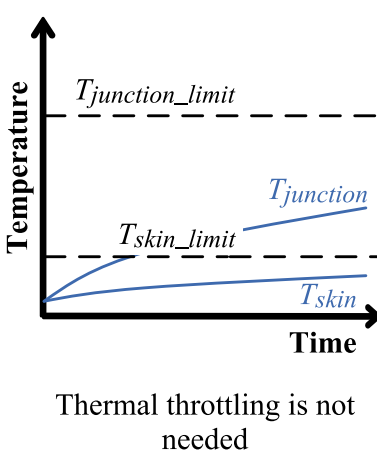

(a)

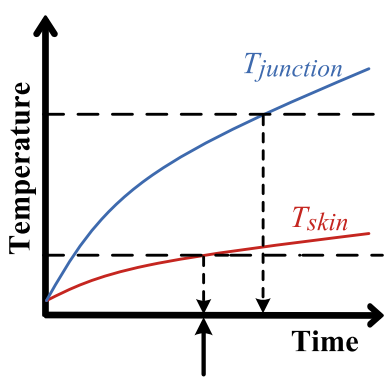

Thermal throttling started by $T_{\text {skin }}$

(b)

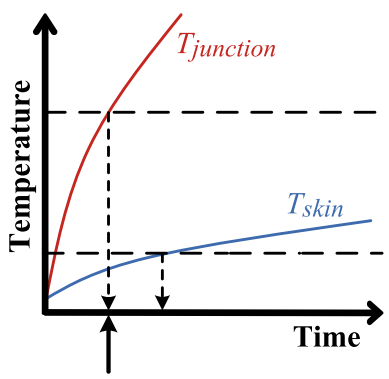

Thermal throttling started by $T_{\text {junction }}$

(c)

Fig. 1. Thermal throttling of junction and skin temperatures over different AP power consumption levels. (a) Low power, (b) Moderate power, (c) High power.

thermal properties between the junction and skin adaptively, according to the varying thermal margins for junction and skin during AP operation. Thermal margin means the remaining time before arriving at the temperature limit of the junction and skin, respectively.

\section{Adaptive thermal property control}

Fig. 2 shows a cross-section schematic and an equivalent RC-thermal network model for a mobile device. The temperature profiles of the junction and skin due to thermal resistance and AP power consumption are calculated using the simplified equivalent RC thermal model of the mobile device shown in Fig. 2(b) [6]. $P_{A P}$ is the AP power consumption. $R_{t h_{-} f}$ is the thermal resistance between junction and front skin.

The state transition equation, which is obtained by applying Kirchhoff's current law (KCL) and Kirchhoff's voltage law (KVL) to each temperature node in Fig. 2(b), is given by (1). $C, G, T$, and $P$ matrices in (1) indicate the thermal capacitance, thermal conductance, temperature difference between nodes, and heat source power, as shown in (2), (3), (4), and (5), respectively.

$$
\begin{gathered}
C \times \frac{d T}{d t}=G \times T+P \\
C=\left[\begin{array}{cccc}
-C_{t h_{-} f} & C_{t h_{a} a i r_{-} f} & 0 & 0 \\
0 & 0 & -C_{t h_{-} b} & C_{t h_{-} a i r_{-} b} \\
C_{t h_{-} f} & 0 & C_{t h_{-} b} & 0 \\
0 & 0 & 0 & 0
\end{array}\right] \\
G=\left[\begin{array}{cccc}
\frac{1}{R_{t h_{-} f}} & -\frac{1}{R_{t h \_a i r_{-} f}} & 0 & 0 \\
0 & 0 & \frac{1}{R_{t h_{-} b}} & -\frac{1}{R_{t h_{-} a i r_{-} b}} \\
-\frac{1}{R_{t h_{-} f}} & 0 & -\frac{1}{R_{t h_{-} b}} & 0 \\
1 & 1 & -1 & -1
\end{array}\right]
\end{gathered}
$$




$$
\begin{gathered}
T=\left[\begin{array}{c}
T_{\text {junction to skin_front }} \\
T_{\text {skin_front to ambient }} \\
T_{\text {junction to skin_back }} \\
T_{\text {skin_back to ambient }}
\end{array}\right] \\
P=\left[\begin{array}{c}
0 \\
0 \\
P_{A P} \\
0
\end{array}\right]
\end{gathered}
$$

The temperature change over time can be calculated by using the finite-difference method based on (1), and we can plot the temperature limit arrival time of the junction and skin according to power $P_{A P}$ and thermal resistance $R_{t h_{-} f}$, as shown in Fig. 3. The solid line denotes the time at which the junction temperature reaches the limit according to $R_{t h_{-} f}$, and the dotted line indicates the time at which the skin temperature reaches the limit according to $R_{t h_{-} f}$ at $4 \mathrm{~W}, 6 \mathrm{~W}$, and $9 \mathrm{~W}$, respectively. The combined cooling capability is decided by the minimum arrival time between junction and skin at each power [2]. Therefore, the thermal resistance at the black point crossed by the solid line and the dotted line will be the ideal $R_{t h_{-} f}$ at each power. We can prevent a false early thermal throttling by adjusting $R_{t h_{-} f}$ according to power.

Fig. 4 shows a pseudo-code of the ATPC. The aim of the ATPC algorithm is to accurately predict the thermal throttling start time considering the temperature limit of either the junction or skin, and adaptively control the thermal resistance to minimize performance degradation caused by false early thermal throttling.

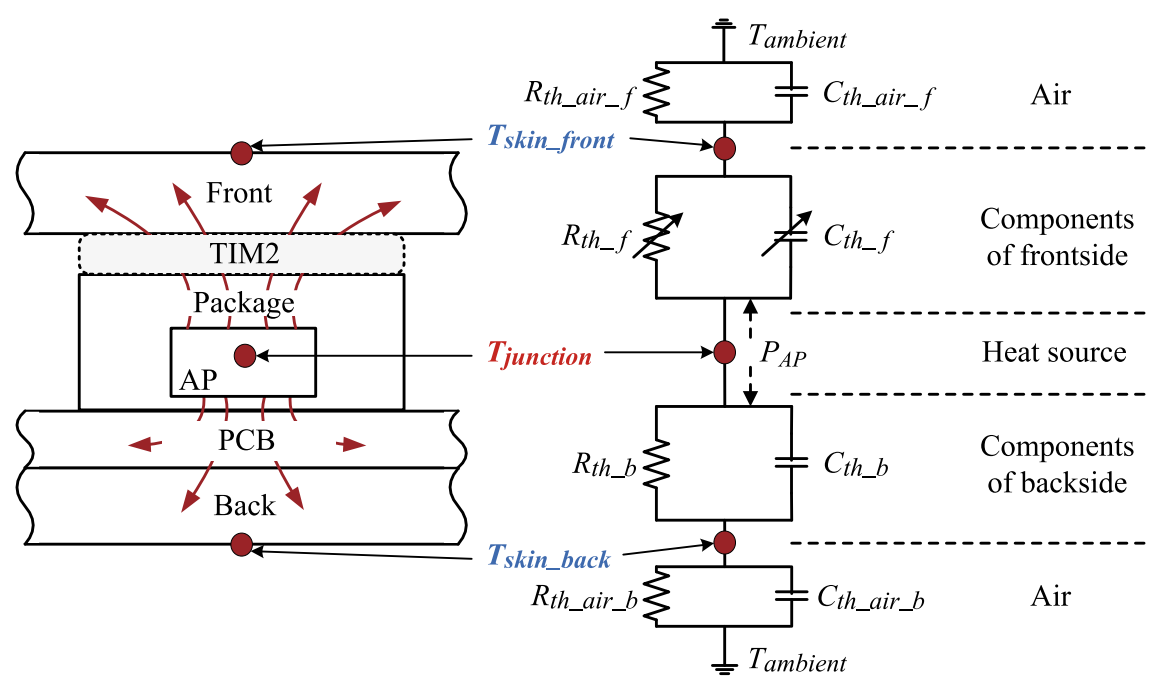

(a)

(b)

Fig. 2. Simplified equivalent thermal model of a mobile device. (a) Cross-section schematic, (b) Equivalent RC thermal model. 


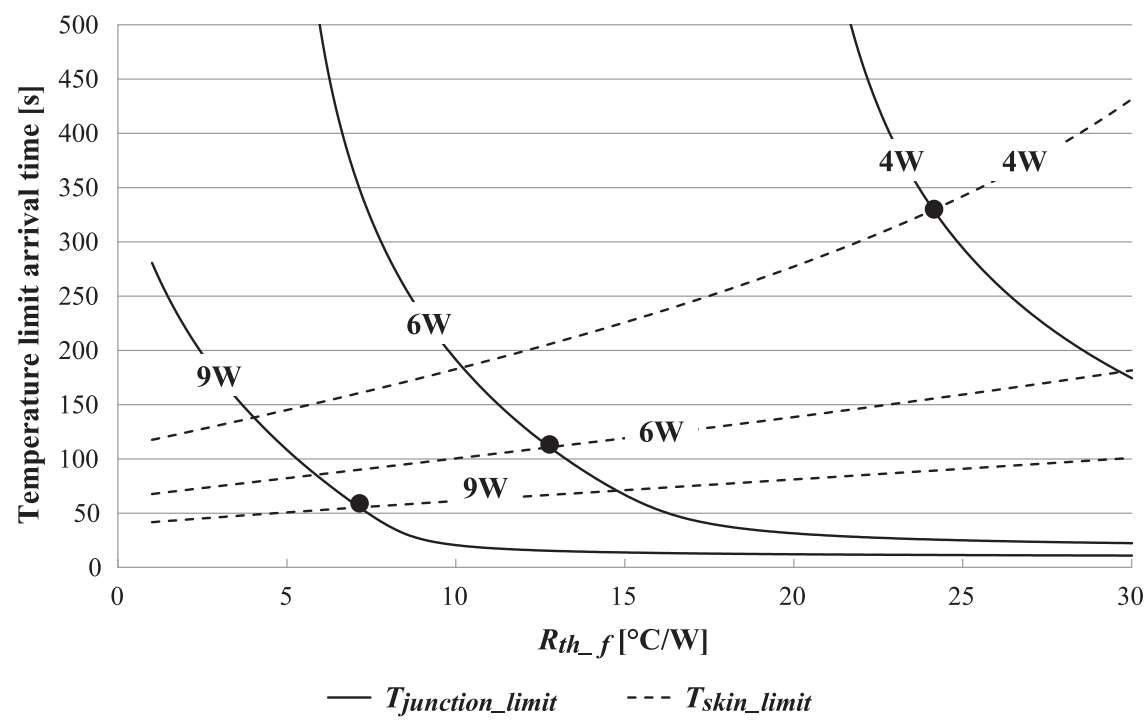

Fig. 3. Ideal thermal resistance at each power.

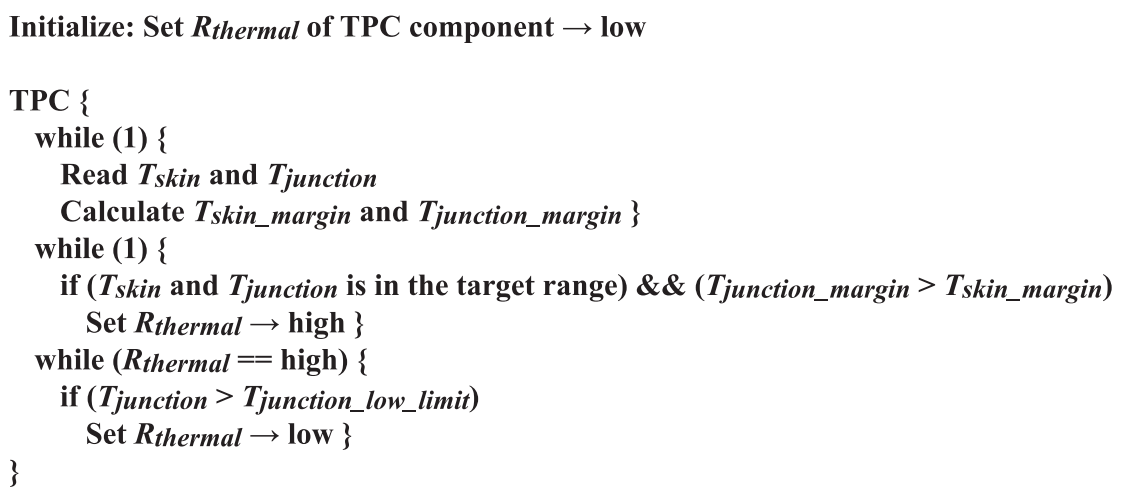

Fig. 4. Basic pseudo-code of the ATPC.

\section{Simulation results and discussion}

The ATPC technique was implemented in Python, and integrated into an IntelDocea power simulator (iDPS) for system-level power-temperature co-simulation [7]. Our thermal model is based on one of the latest Samsung smartphones, whose AP contains eight CPU cores fabricated using a Samsung 10-nm CMOS process, and packaged in an interposer package on package (I-POP) with 4 GB LPDDR4x DRAM [8].

Fig. 5 shows the example of the increased cooling capacity achieved due to ATPC by plotting temperature versus time at an AP power of $4 \mathrm{~W}$. When $T_{\text {skin }}$ reaches $39^{\circ} \mathrm{C}$, which is a predefined threshold, and the junction thermal margin is bigger than the skin thermal margin, the thermal conductivity changes from $3 \mathrm{~W} / \mathrm{m} \cdot \mathrm{K}$ to $0.3 \mathrm{~W} / \mathrm{m} \cdot \mathrm{K}$. As a result, the temperature profile changes to dotted lines at $326.5 \mathrm{~s}$ and we can adjust false early thermal throttling from $393 \mathrm{~s}$ (square) to $478 \mathrm{~s}$ (circle), whereas the junction temperature did not surpass its threshold temperature of $85^{\circ} \mathrm{C}$ (upper dotted red line). 


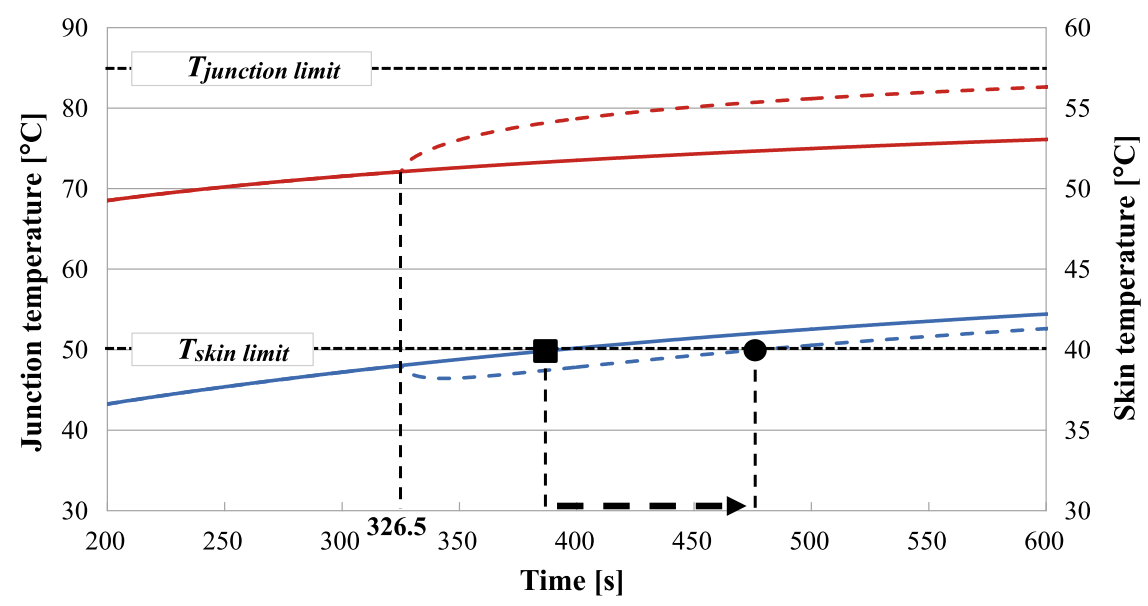

—Tjunction@k_fix_3 - - Tjunction@ATPC - Tskin@

Fig. 5. Temperature profile change at $4 \mathrm{~W}$ by ATPC.

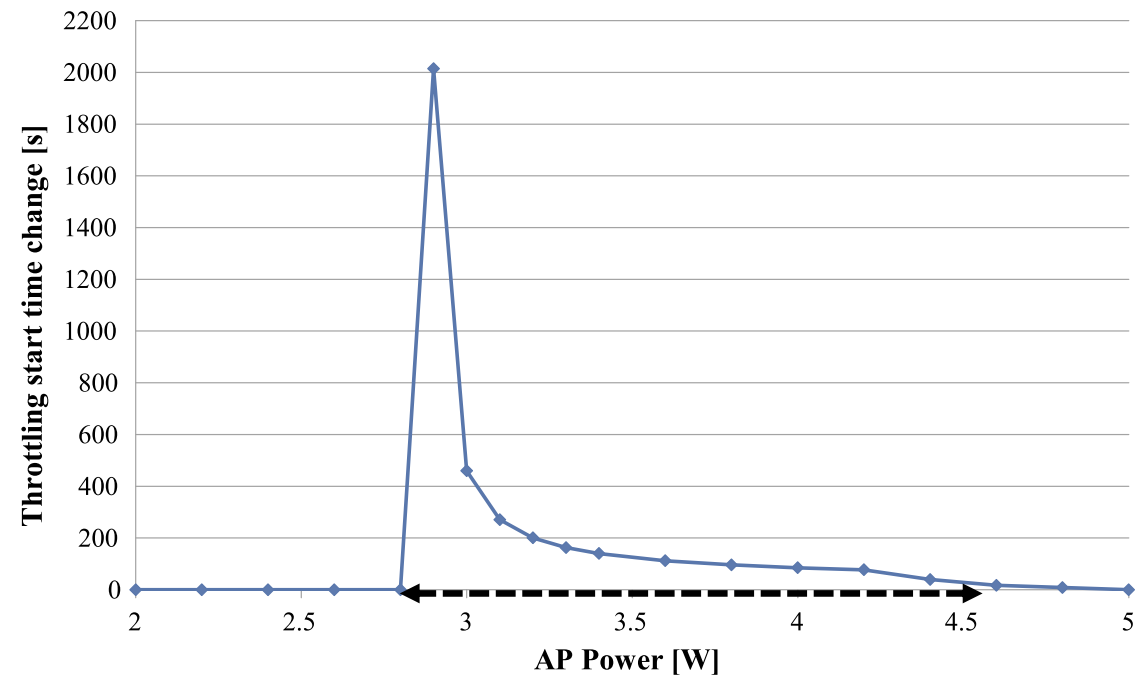

Fig. 6. Performance gain by ATPC.

Fig. 6 shows the change in the start time of thermal throttling due to ATPC in the AP power range of 2.9-4.6 W. ATPC can prevent false early thermal throttling up to $2040 \mathrm{~s}$ (34 min).

From the perspective of the AP power budget gain, up to $7 \%$ more power can be used than in the case of no ATPC with the same operating time without thermal throttling. This means that the ATPC technique achieves a performance improvement of approximately $7 \%$.

\section{Conclusion}

We presented an ATPC technique that controls the thermal properties between the junction and skin adaptively, according to the thermal margin of the junction and skin. The simulation results showed that ATPC can be used to prevent false early thermal throttling up to $34 \mathrm{~min}$ in the AP power consumption range of 2.9-4.6 W. The ATPC technique defied the stereotype that the material property of the mobile device is fixed during the design time and cannot be changed. 
The ATPC component for the mobile industry can be produced by applying advanced material and microelectromechanical systems (MEMS) technology, which is well-known in the research field today $[9,10,11]$.

\section{Acknowledgments}

This work was supported by the MOTIE (Ministry of Trade, Industry \& Energy (10080594) and KSRC (Korea Semiconductor Research Consortium) support program for the development of the future semiconductor device and by the IT R\&D Program of MSIP/IITP (2016-0-00088).

The authors would like to express gratitude to Prof. Yogendra Joshi at Georgia Institute of Technology for his guidance on thermal meta-materials. 